\title{
Corneal Collagen Cross-linking in a Prepubescent 10-Year-Old Girl with Aggressive Keratoconus
}

\author{
${ }^{1}$ Marco Abbondanza, ${ }^{2}$ Margherita Guidobaldi
}

\begin{abstract}
Keratoconus $(\mathrm{KC})$ is a corneal ectatic disease that results in bilateral and asymmetrical corneal distortion, altered refractive powers and reduced vision. In 20 to $25 \%$ of cases, corneal transplantation may be required, although a number of conservative procedures are available. We report a case of an aggressive stage II keratoconus in a prepubescent 10 -year-old girl, successfully treated with corneal collagen cross-linking $(\mathrm{CXL})$ with epithelium removal.
\end{abstract}

Keywords: Cornea, Corneal collagen cross-linking, Keratoconus, Pediatric.

How to cite this article: Abbondanza M, Guidobaldi M. Corneal Collagen Cross-linking in a Prepubescent 10-YearOld Girl with Aggressive Keratoconus. Int J Kerat Ect Cor Dis 2015;4(2):63-65.

\section{Source of support: Nil \\ Conflict of interest: None}

\section{INTRODUCTION}

Keratoconus (KC) is a corneal ectatic disease characterized by the gradual loss of thickness and change into a more conical shape of the corneal stroma. This leads to irregular astigmatism and subsequent decrease in visual acuity $^{1}$ while, in 20 to $25 \%$ of cases, corneal transplantation may be required. The clinical onset is generally bilateral and occurs at a young age, usually in the second decade of life.

Conservative surgical techniques, aimed at avoiding the need for a corneal transplantation, include asymmetric radial keratotomy $(\mathrm{ARK}),{ }^{2}$ mini asymmetric radial keratotomy (MARK), ${ }^{3}$ circular keratotomy $(\mathrm{CK})^{4}$ and intrastromal corneal ring segments (ICRS). ${ }^{5}$

Another original technique recently developed is corneal collagen cross-linking (CXL), a procedure that directly addresses the intrinsic structural weakness of the cornea by reinforcing the corneal stroma, thus stopping keratoconus progression. ${ }^{6}$ Corneal collagen cross-

\footnotetext{
${ }^{1}$ Head, ${ }^{2}$ Orthoptist

1,2Department of Surgery, Abbondanza Eye Center, RomeMilan, Italy

Corresponding Author: Marco Abbondanza, Head Department of Surgery, Abbondanza Eye Center, Rome-Milan Italy, e-mail: info@abbondanza.org
}

linking consists in the removal of corneal epithelium, followed by the local application of a riboflavin solution and by subsequent UV-A irradiation, a technique firstly developed by surgeons at the Dresden Clinic, Germany.

We have successfully performed CXL since 2005, reporting both a mean refractive improvement of $1,2 \mathrm{D}$ and a stabilization of the disease following the intervention, ${ }^{7}$ alone or combined with other refractive procedures, including MARK, photorefractive keratectomy (PRK) and phakic intraocular lens (PIOL) implantation. This case report, however, focuses on the outcomes of a single CXL treatment in a prepubescent 10 -year-old girl with an aggressive stage II keratoconus.

\section{CASE REPORT}

In May 2014, a 10-year-old girl presented with a bilateral keratoconus diagnosed 45 days before, ocular history included 6 years of vernal keratoconjunctivitis. The first diagnosis of hyperopia and astigmatism has been advised 4 years before by her optician.

The patient underwent a thorough clinical examination prior to the intervention, which included measurement of uncorrected (UCVA) and best corrected visual acuity (BCVA), computerized corneal topography, axial biometry, pachymetry, endothelial cell count, keratometry, Scheimpflug camera corneal analysis and slit lamp examination.

Corrected distance visual acuity (CDVA) was 20/25 in the left eye with $+0.50 /-1.50 \times 160$ and $20 / 32$ in the right eye with $-2.50 \times 5$. Central keratometry $(K)$ values were $45 \times 48$ Dioptres (D) in the left eye and $45.5 \times 52.5 \mathrm{D}$ in the right eye. $\mathrm{K}_{\max }$ was $55.2 \mathrm{D}$ in the right eye, showing a dramatic worsening compared to prior examinations, when $\mathrm{K}_{\max }$ values in the left eye and the right eye were comparable. Central pachimetry (Pentacam-Oculus Optikgerate $\mathrm{GmbH}$, Wetzlar) was $534 \mu \mathrm{m}$ in the right eye and $561 \mu \mathrm{m}$ in the left eye. Thinnest pachymetry was 473 $\mu \mathrm{m}$ in the right eye (Fig. 1).

Given the rapid progression of the disease, we performed the CXL treatment in the right eye according to the Dresden protocol (epi-off), with benoxinate chloride $0.4 \%$ drops as a topical anesthesia. Removal of corneal epithelium was performed using an ophthalmic scalpel (MicroFeather; Feather Safety Razor Co, Ltd, Osaka, 


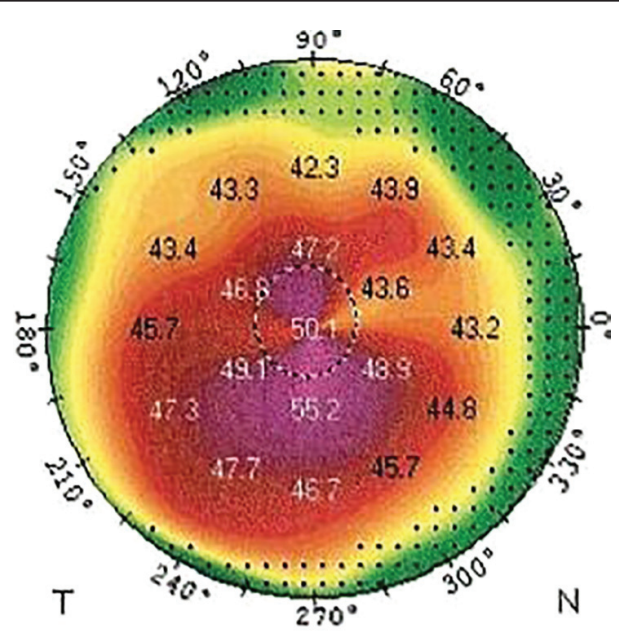

Fig. 1: Corneal topography of the right eye before the intervention, $\mathrm{K}_{\max }$ value was $55.2 \mathrm{D}$

Japan) and a surgical microscope. Eyelids were kept open using surgical forceps during the procedure.

Riboflavin eye drops (B2 vitamin) were applied on the corneal stroma 30 minutes before UV-A application 10 times at 3 minute intervals, then riboflavin was applied six times at 5 minute intervals during UV-A application, with a total UV-A exposure time of 30 minutes. The UV-A source used was the UV-X illumination system version 1000, from IROC AG, Switzerland.

After treatment, a contact lens was placed and topical antibiotics and non-steroidal anti-inflammatory drops were prescribed. Clinical examination was performed on the following day. The contact lens was removed 3 days following the treatment and corneal epithelial healing was checked.

Three months after CXL, K values were $45.75 \times 52 \mathrm{D}$ and $\mathrm{K}_{\max }$ was $55 \mathrm{D}$. Six months after CXL, CDVA was 20/32, K values were $45.5 \times 52$ and $\mathrm{K}_{\max }$ decreased to 53.9 D (Fig. 2 and Table 1).

The patient's left eye has been regularly monitored with thorough clinical examinations, which showed a stable condition and no need for CXL.

\section{DISCUSSION}

Corneal collagen cross-linking with epithelium removal is effective in reducing the progression of keratoconus, along with a painless and safe protocol for adult patients. For this reason, CXL has been recently applied to children as well.

Ophthalmic case studies in pediatric and adolescent patients with keratoconus have shown that, following $\mathrm{CXL}, \mathrm{KC}$ is stabilized with a significant visual and topo-

Table 1: Pre- and post-CXL values, 6 months after the intervention

\begin{tabular}{llllllll}
\hline & $I H A$ & $I H D$ & $I S V$ & $I V A$ & $K I$ & $C K I$ & $K_{\max }$ \\
\hline Pre-CXL & 44.7 & 0.054 & 0.061 & 0.061 & 1.19 & 1.05 & 55.2 \\
Post-CXL & 44.8 & 0.051 & 0.061 & 0.047 & 1.18 & 1.05 & 53.9 \\
\hline
\end{tabular}

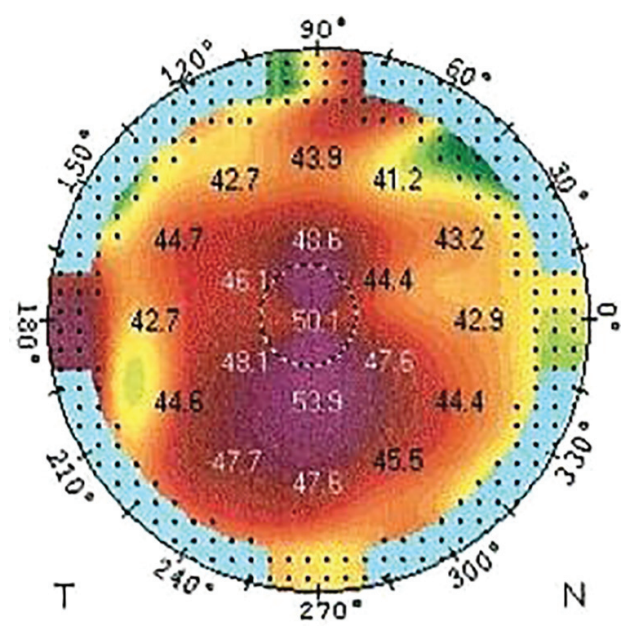

Fig. 2: Corneal topography of the right eye 6 months after CXL, $\mathrm{K}_{\max }$ and astigmatism improved

graphic improvement. Due to an increasing awareness of the disease, a number of studies demonstrate that pediatric keratoconus is now often diagnosed and treated with success. While the number of children and adolescents affected by keratoconus varies depending on the kind of clinical trial, two studies show that it ranges between $29^{8}$ and $38 \%{ }^{9}$ of all treated keratoconus patients. This parasurgical treatment is safe and there is no visually significant risk, ${ }^{10-12}$ although one study suggests that the halting of the disease might not be as long lasting as in adults. ${ }^{12}$ It is worth pointing out, however, that we never had to repeat the treatment in our 10-year experience with CXL, even when dealing with pediatric keratoconus.

Pediatric keratoconus shows a higher incidence rate and a more rapid progression compared to adult keratoconus, ${ }^{12}$ notwithstanding the fact that the disease is most frequently diagnosed after adolescence. The progressive visual impairment influences the social life as well as the educational development of affected children. An early diagnosis of keratoconus, by adoption of adequate screening procedures, is therefore fundamental.

In our case, the diagnosis of keratoconus was early and its treatment with 'epi-off' CXL was timely. Manifest refraction remained stable during follow-up, as the $\mathrm{K} 2$ value, while $\mathrm{K}_{\max }$ value improved. In our last examination, 6 months after CXL, the apex of the cone had decreased by $1.3 \mathrm{D}$ and there were no complications, thus showing a stabilization of keratoconus as well as a visual improvement, despite the aggressiveness of the disease. These data let us hope for new improvements in the near future.

\section{REFERENCES}

1. Rabinowitz YS. Keratoconus. Survey of Ophthalmology 1998; 42(4):297-319.

2. Abbondanza M. Asymmetric radial keratotomy for the correction of keratoconus. J Refract Surg 1997;13(3):302-307. 
3. Abbondanza M. Mini Cheratotomia Radiale Asimmetrica (Mini ARK) per la correzione chirurgica del cheratocono in fase iniziale, nell'ipermetropia e nelle miopie lievi. Esperienze 1997;12(1):21-24.

4. Krumeich JH, Keziran GH. Circular keratotomy to reduce astigmatism and improve vision in stage I and II keratoconus. J Refract Surg 2009;25(4):357-365.

5. Chan Colin CK, Sharma M, Boxer Wachler BS. The effect of inferior segment intacs with and without corneal collagen crosslinking with riboflavin (C3-R). J Catar Refract Surg 2007;33(1):75-80.

6. Wollensak G. Crosslinking treatment of progressive keratoconus: new hope. Current Opinion in Ophthalmology 2006; 17(4):356-360.

7. Abbondanza M, Abdolrahimzadeh B, Zuppardo M. Refractive changes following CXL. Cataract and Refractive Surgery Today Europe 2009;4(7):33-38.
8. Caporossi A, Mazzotta C, Baiocchi S, Caporossi T, Denaro R. Age-related long-term functional results after riboflavin UV A corneal cross-linking. J Ophthalmol 2011;2011:608041.

9. Al Suhaibani AH, Al-Rajhi AA, Al-Motowa S, Wagoner MD. Inverse relationship between age and severity and sequelae of acute corneal hydrops associated with keratoconus. Br J Ophthalmol 2007;91(7):984-985.

10. Barbara R, Pikkel J, Garzozi H, Barbara A. Collagen crosslinking and keratoconus in pediatric patients. Int J Kerato Ectatic Corneal Dis 2012;1(1):57-60.

11. Chatzis N, Hafezi F. Progression of keratoconus and efficacy of pediatric (corrected) corneal collagen cross-linking in children and adolescents. J Refract Surg 2012;28(11): 753-758.

12. Léoni-MespliéS, Mortemousque B, Touboul D, Malet F, Praud D, Mesplié N, et al. Scalability and severity of keratoconus in children. Am J Ophthalmol 2012;154(1):56-62. 